\title{
Percentage of Blastulation on the Number and Function of Meta- phase II Oocytes
}

\author{
Martha L. Chadid ${ }^{1}$, Jorge Carpio ${ }^{1}$, Pedro Valdivieso ${ }^{1}$, Milton Zambrano ${ }^{1}$, Javier García-Ferreyra ${ }^{1}$, Pedro Valdivie- \\ so-Mejía ${ }^{1}$
}

${ }^{1}$ Unidad de Fertilidad - Hospital Alcívar - Guayaquil, Ecuador

\begin{abstract}
Objective: Establish the number of metaphase II oocytes that are needed for optimum blastulation rate and to achieve a better rate of gestation.

Methods: Retrospective study. Women diagnosed with infertility. There were 110 women submitted to IVF/ICSI, excluding third day transfers and those with canceled cycles. After controlled ovarian stimulation, cultivation continued to blastocysts.

Results: Average age of the patients: 33.41 . IVF technique: 73 patients, $66.4 \%$; ICSI: 37 patients, $33.6 \%$. Pregnancy: gestation: $57.3 \%$; Non-gestation: $42.7 \%$. Mean number of oocytes in metaphase II: 7.5.

Conclusion: The gestation rate coincides with the center's results $(50-60 \%)$, reached with 7 mature oocytes. Mean blastulation rate was $39.2 \%$.
\end{abstract}

Keywords: IVF (In Vitro Fertilization), ICSI (Intracytoplasmic Sperm Injection).

\section{INTRODUCTION}

Proper evaluation of embryo quality is crucial to succeed in an IVF program. In most of the assisted reproduction clinics this assessment is mainly based on morphological evaluation of embryos upon division.

Blastulation is the process by which the blastocyst is formed; constituting the embryonic stage created by a sphere of blastomeres in which volume and position depend on yolk quantity and distribution (Gardner \& Schoolcraft, 1999).

After 5 days, the embryo passes from the morula to the blastocyst stage - formed by a mass of cells.

It is then, released from the zona pellucida and is set to install inside the blastocele (or embryoblast: group of compacted cells), where it is covered by an epithelial layer called trophectoderm (which will originate extra-embryonic organs: placenta and amniotic membranes).

After 6 days of fertilization, the blastocyst significantly increases in size in the uterus, continuing with its proper development. Embryo quality is assessed by its morphology and development.

Division is an important process, likewise the time period between each of the divisions. After arriving at the 8-cell stage, the blastomere begins to adhere due to increased intercellular junctions; it is the beginning of compaction. On the fifth day of development, after cell compaction, a cavity called blastocele begins to develop and cell differentiation ensues. Blastocyst expansion degree and duration are important predictors of implantation.

Not all embryos are able to reach the blastocyst stage. It has been reported that between $40 \%$ and $60 \%$ of in vitro fertilized oocytes reach this stage, and this ability is directly related to the embryo morphology at an earlier stage.

There are certain clinical factors related to high rates of blastulation, such as young age with previous gestation, conventional in vitro fertilization and low dose gonadotropin used in the stimulation (Thomas et al., 2010).
Most embryos with proper development reach the blastocyst stage on the fifth day of development (112-120 hours post insemination), but some have a slower development - differentiating on the sixth day (136-140 hours post insemination). The implantation rate of these embryos is lower, but not negligible (Seli et al., 2004).

One of the priorities in assisted reproduction techniques is to reduce the incidence of multiple pregnancies and higher number of sacs (more than 2 fetal sacs), and the complications they ensue, without affecting implantation rates and RNV (Nyboe-Andersen et al., 2008).

Recent advances in cell culture technology helped change the practice of IVF from embryo transfer in the early cleavage stage to transfers in the blastocyst stage (Kissin et al., 2005). The main reason for culturing blastocysts is to enhance uterine and embryonic synchronicity, and the selection of viable embryos, resulting in high implantation rates (Pandian et al., 2005).

\section{MATERIAL AND METHODS}

This is a retrospective review of 110 IVF-ICSI cycles, excluding patients transferred on day 3 and canceled cycles.

The cycles were performed during one year from March 2013 to 2014 in the Fertility Unit of Alcivar's Hospital (Guayaquil, Ecuador). In all the cases, the patients were submitted to controlled ovarian stimulation using $\mathrm{GnRH}$ agonist or antagonist protocols.

During these cycles, gonadotropin stimulation was carried out until at least two follicles reached a diameter of 17 to $20 \mathrm{~mm}$. Oocyte collection was monitored using a trans-vaginal guided echo device, 36 hours after the administration of $250 \mathrm{mg}$ of recombinant hCG.

After oocyte harvesting, fertilization was achieved by conventional insemination or by Intracytoplasmic sperm injection (ICSI) in cases of a severe male dysfunction.

The development in culture continues to blastocyst, the embryos will change on Day 3 in fresh Global medium $10 \%$ SSS and undergo embryonic development evaluation on days 3 and 5 . Blastocysts with better morphology are transferred and the others are vitrified.

We assessed the rate of blastocyst formation and the relationship of blastulation quality according to the number of oocytes harvested in Metaphase II. The main issues were: metaphase II oocytes, patient's age, pregnancy, and IVF-ICSI.

\section{RESULTS}

The information was analyzed with the SPSS 20.0, using the statistical average \pm standard deviation for continuous variables and qualitative percentage. We used the Chi square analysis.

Concerning gestational age, the subjects were divided into 2 groups:

$1^{\text {st }}$ No gestation: 35.8 ; SD: 34.5

$2^{\text {nd }}$ Gestation: 33.7; SD: 32.5

Age is an important factor in pregnancy, between 23- 
35 years of age there is a greater gestation percentage, with 0.001 of significance at the $95 \%$ confidence interval.

In our study, we analyzed a total of 736 embryos in normal cell division phase. These embryos were from 110 IVF cycles carried out in 110 patients. All of the embryos were analyzed.

Blastulation average rate was $39.2 \%$ for all embryos.

IVF: 73 patients, $66.4 \%$; ICSI: 37 patients, $33.6 \%$ (Figure 1).

\section{FIV-ICSI}

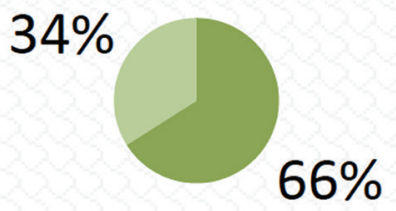

\section{FIV ICSI}

Figure 1: FIV $66.4 \%$; ICSI: $33.6 \%$.

Rate of Pregnancy (Figure 2): Pregnancy: 57.3\%; No pregnancy: $42.7 \%$.

Rate of oocytes in metaphase II: Mean of 7.5.

In the group of patients that received 5 mature oocytes, the pregnancy rate was $39 \%$. When they received 6 mature oocytes, the pregnancy rate went up to $48 \%$.

With 7 mature oocytes we obtained a pregnancy rate of $54 \%$.

With 8 mature oocytes our pregnancy rate was $58 \%$.

With 9 oocytes, the pregnancy rate was $58.6 \%$.

With 10 mature oocytes, the pregnancy rate was $57.36 \%$.

With more than 11 oocytes, the pregnancy rate was $50 \%$. A statistically significant $P$-value of 0.002 . Figure 3 shows this results.

\section{Pregnancy}

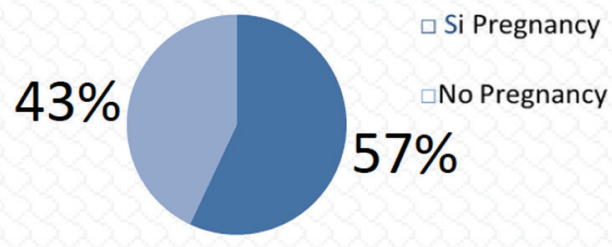

Figure 2: Relationship between metaphase II oocytes and pregnancy.

\section{DISCUSSION}

According to these results, the pregnancy rate we achieved was $50-60 \%$ with 7 metaphase II oocytes.

Pregnancy rates were very similar above this number of oocytes.

Age is a relevant factor in pregnancy, the highest percentage was found in subjects between 23-35 years of age. Blastocyst transfer had a better synchronization at an endometrial level, because when compaction begins, the embryos reach the endometrial cavity; in addition, nutritional needs are different at this stage. Improper transfer in relation to the stage of embryonic development can lead

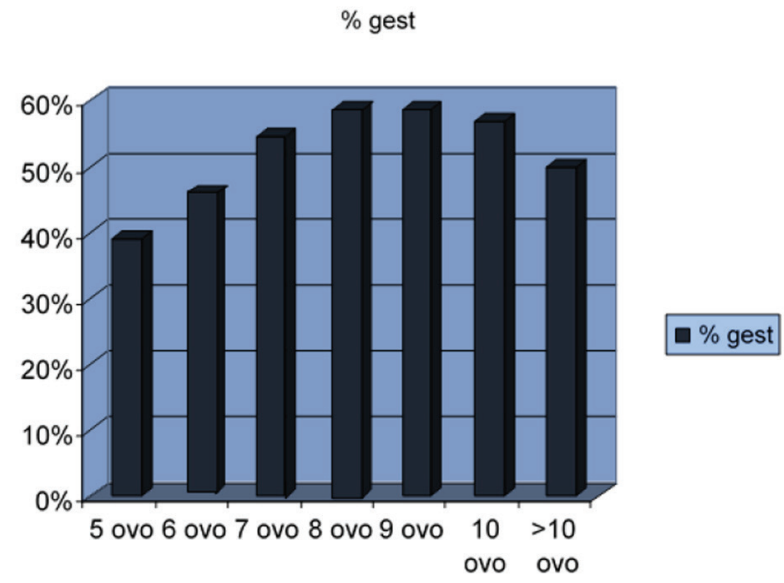

Figure 3: Relationship between metaphase II oocytes and pregnancy.

to metabolic stress, which could adversely affect embryo viability (Sadler \& Langman, 2004).

Implantation occurs from day 5 to 7 after fertilization, known as "implantation window" (Larsen, 2003). Many studies show that implantation also occurs when there are three days of asynchronicity, but the ideal time would be a maximum of 1 day of asynchronicity separating embryo and endometrium (Núñez de Castro, 2008)

It has also been shown that uterine contractility decreases as the days go by and the endometrium at 5 days post-puncture seems to be optimum for embryo implantation, with an implantation window similar to that of natural conception (Fanchin et al., 2001).

\section{CONCLUSIONS}

- Pregnancy rate matching with the center's results (50-60\%) were reached with 7 mature oocytes.

- Above this number of oocytes in metaphase II, the results in pregnancy rates were very similar to those from other reports.

- Age is an influential factor in pregnancy; subjects between 23-35 years of age have a higher pregnancy rate. We achieved a blastocyst culture, which is the main goal to provide a healthy and normal newborn to the infertile couple. With a blastulation rate of $39.2 \%$.

\section{CONFLICT OF INTERESTS}

No conflict of interest have been declared.

\section{Corresponding author:}

Martha Luna Chadid

Fertility Unit - Alcivar Hospital

Guayaquil, Ecuador

E-mail: mluna_chadid@hotmail.com

\section{REFERENCES}

Fanchin R, Ayoubi J, Righini C, Olivennes F, Schönauer LM, Frydman R. Uterine contractility decreases at the time of blastocyst transfers. Hum Reprod 2001; 16:1115-9.

Gardner DK, Schoolcraft WB. In vitro culture of human blastocyst. In: Toward Reproductive Certainty: Fertility and Genetics Beyond 1999. Jansen R, Mortimer D, ed. London, UK: Parthenon Publishing Group, 1999; 378-88.

Kissin DM, Schieve LA, Reynolds MA. Multiple-birth risk associated with IVF and extended embryo culture: USA, 2001. Hum Reprod 2005; 20:2215-23. 
Larsen WJ, ed.. Embriología Humana. Madrid: Editorial Elselvier; 2003.

Núñez de Castro I. De la dignidad del embrión. Reflexiones en torno a la vida humana naciente. Madrid: Universidad Pontificia Comillas; 2008.

Nyboe-Andersen A, Goossens V, Ferraretti AP, Bhattacharya S, Felberbaum R, de Mouzon J, Nygren KG; The European IVF-Monitoring (EIM) Consortium for the European Society of Human Reproduction Embryology (ESHRE). Assisted reproductive technology in Europe, 2004: results generated from European registers by ESHRE. Hum Reprod 2008; 23:756-71.

Pandian Z, Templeton A, Serour G, Bhattacharya S. Num- ber of embryos for transfer after IVF and ICSI: a Cochrane review. Hum Reprod 2005; 20:2681-87.

Sadler TW, Langman J, eds. Embriología Médica con orientación clínica. Madrid: Editorial Médica Panamericana, 2004.

Seli E, Gardner DK, Schoolcraft WB, Moffatt O, Sakkas D. Extent of nuclear DNA damage in ejaculate spermatozoa impacts on blastocyst development after in vitro fertilization. Fertil Steril 2004; 82:378-83.

Thomas MR, Sparks AE, Ryan GL, Van Voorhis BJ. Clinical predictors of human blastocyst formation and pregnancy after extended embryo culture and transfer. Fertil Steril 2010; 94:543-8. 